\title{
Verification of Accuracy Improvement for CoMPACT Monitor Due to Suboptimal Inter-probe Time
}

\author{
Kohei Watabe Yudai Honma and Masaki Aida / Graduate School of System Design \\ dept. Management Systems Engineering \\ Tokyo Metropolitan University, \\ Hino-shi, Japan \\ \{watabe-kouhei, yudai, maida\}@sd.tmu.ac.jp
}

\begin{abstract}
CoMPACT monitor gives the one-way delay distribution of a target flow by using a transformation of one-way delay data obtained by active measurement. The transformation can be determined from passively monitored traffic data for the target flow of the measurement. In a recent study, it was reported that by using an inter-probe time that has a Gamma distribution can improve the accuracy of simple active measurement. In this study, we show that CoMPACT monitor is improved in accuracy by the application of new active measurement.
\end{abstract}

\section{Introduction}

Today the Internet is used not only as a private tool but also a business tool, and plays an important role as infrastructure. The traffic generated by emerging new applications, including telephony and live video in addition to the traditional e-mail and web browsing, exhibits complex characteristics. Moreover, the quality of service (QoS) required by various applications is also quite different. When new applications are developed in the future, the QoS requirements will be changed even more.

In order to meet such varied requirements for network control, we need a measurement technology to produce detailed QoS information. Measuring the QoS for each of multiple flows (e.g., users, applications, or organizations) is important since these are used as key parameters in service level agreements (SLAs) between an Internet service provider (ISP) and users. One-way packet delay is one of the most important QoS metrics. This paper focuses on the measurement of one-way delay for each flow.

Conventional means of measuring network performance and QoS can be classified into two types: passive and active measurements.

Passive measurement monitors the target user packet directly, by capturing the packets, including the target information. Passive measurement is used to measure the volume of traffic, one-way delay, round-trip time (RTT), loss, etc. and can get any desired information about the traffic since it observes the actual traffic. Passive measurement can be categorized into two-point monitoring with data-matching processes (to measure one-way delay etc.) and one-point monitoring (to measure volume of traffic etc.).

Passive measurement has the advantage of accuracy. However if we perform passive measurement in large-scale networks, the number of monitored packets is enormous and network resources are wasted by gathering the monitored data at a data center. Moreover, in order to measure delay, it is necessary to determine the difference in arrival time of a particular packet at different points in the network. This requires searching for the same packet pairs monitored at the different points in the monitored packet data. This packet matching process lacks scalability, so passive measurement lacks scalability.

Active measurement monitors QoS by injecting probe packets into a network path and monitoring them. Active measurement can be used to measure one-way delay, RTT, loss, etc. It cannot obtain the per-flow QoS, though it is easy for the end user to carry out. Unfortunately, the QoS data obtained by active measurement does not represent the QoS for user packets, but only QoS for the probe packets.

By complementary use of the advantages of active and passive measurements, the authors propose a new technique of scalable measurement called change-of-measure-based passive/active monitoring (CoMPACT monitor) to measure per-flow QoS [1], [2].

The idea of CoMPACT monitor is as follows. The direct measurement of the one-way delay distribution of the target flow by passive measurement is difficult due to the scalability problem. So, we try to obtain the one-way delay distribution of the target flow by using a transformation of one-way delay data obtained by active measurement. The transformation can be determined by passively monitored traffic data for the target flow of the measurement. The problem of scalability does not arise, because the volume of traffic can be measured by one-point passive measurement without requiring data-matching processes.

We have believed Poisson arrivals (intervals according to exponential distribution) is appropriate to a policy of probe packets arrivals since we can apply PASTA (Poisson Arrivals 
See Time Averages) property to it.

However, recent work [3] indicates that many distributions exist that are more accurate than an exponential distribution if a non-intrusive context (ignoring the effect of probe packets) can be assumed. Moreover, according to [3] we can find a distribution that is suboptimal in accuracy by selecting an inter-probe time according to the parameterized Gamma distribution.

In this study, we have applied this Gamma-probing to the active measurement part of CoMPACT monitor and tried to improve CoMPACT monitor's in accuracy. This paper confirms that Gamma-probing is appropriate when measuring the complementary cumulative distribution function (CDF) of individual flows by simulation.

\section{Summary of CoMPACT monitor}

CoMPACT monitor estimates an empirical QoS for the target flow by converting observed values of network performance at timing of probe packet arrivals into a measure of the target flow timing. Now, let $V(t)$ denote the network process under observation (e.g. the virtual one-way delay in the network path at time $t$ ), and $X_{k}$ denote a random variable which is observed $V(t)$ with a certain timing (e.g. the timing of user $k$ 's packet arrivals). The probability for $X_{k}$ to exceed $c$ is

$$
\mathrm{P}\left(X_{k}>c\right)=\int 1_{\{x>c\}} \mathrm{d} F_{k}(x)=\mathrm{E}_{F_{k}}\left[1_{\{x>c\}}\right]
$$

where $F_{k}(x)$ is the CDF of $X_{k}$.

If we can directly monitor $X_{k}$, its distribution can be estimated by $\sum_{n=1}^{m} 1_{\left\{X_{k}(n)>c\right\}} / m$, for sufficiently large $m$. Now, let us consider the situation that $X_{k}$ cannot be directly monitored. Let $Y$ denote a random variable that is observed $V(t)$ at a different timing (e.g. timing of probe packet arrivals) independent of $X_{k}$. Then we consider the relationship between $X_{k}$ and $Y$.

Observed values of $X_{k}$ and $Y$ are different if their timing is different, even if they observe a common process $V(t)$. $X_{k}$ and $Y$ can be related by each $\operatorname{CDF} F_{k}(x)$ and $G(y)$, and $\mathrm{P}\left(X_{k}>c\right)$ expressed by measure of $X_{k}$ can be transformed into measure of $Y$ as follows.

$$
\begin{aligned}
\mathrm{P}\left(X_{k}>c\right)=\int 1_{\{x>c\}} \mathrm{d} F_{k}(x) & =\int 1_{\{y>c\}} \frac{\mathrm{d} F_{k}(y)}{\mathrm{d} G(y)} \mathrm{d} G(y) \\
& =\mathrm{E}_{G}\left[1_{\{Y>c\}} \frac{\mathrm{d} F_{k}(Y)}{\mathrm{d} G(Y)}\right]
\end{aligned}
$$

Therefore, $\mathrm{P}\left(X_{k}>c\right)$ can be estimated by

$$
\frac{1}{m} \sum_{n=1}^{m} 1_{\{Y(n)>c\}} \frac{\mathrm{d} F_{k}(Y(n))}{\mathrm{d} G(Y(n))},
$$

where $Y(n)(n=1,2, \cdots, m)$ denote the $n$th observed value. Note that this estimator does not need to monitor the timing of $X_{k}$, if we can get $\mathrm{d} F_{k}(Y(n)) / \mathrm{d} G(Y(n))$.
In the following, we briefly summarizes the mathematical formulation of CoMPACT Monitor [2].

Let $a(t)$ and $V(t)$, respectively, denote the traffic in the target flow at time $t$ and the virtual one-way delay on the path that we want to measure. Considering to measure the empirical one-way delay distribution, the value we want to measure is the ratio to all traffic of the target flow of traffic for which the delay to exceeds $c$, which is given by

$$
\pi(c)=\lim _{t \rightarrow \infty} \frac{\int_{0}^{t} 1_{\{V(s)>c\}} a(s) \mathrm{d} s}{\int_{0}^{t} a(s) \mathrm{d} s}
$$

This can be estimated through $m$ times monitoring by

$$
Z_{m}(c)=\frac{1}{m} \sum_{n=1}^{m} 1_{\left\{V\left(T_{n}\right)>c\right\}} \frac{a\left(T_{n}\right)}{\sum_{l=1}^{m} a\left(T_{l}\right) / m}
$$

for sufficiently large $m$ (see [2] for details), where $T_{n}$ $(n=1,2, \cdots, m)$ denotes the $n$th sampling time, and each time of sampling corresponds to a time of probe packet arrival. Active and one-point passive measurement are used respectively to observe $V\left(T_{n}\right)$ and $a\left(T_{n}\right)$. Note that onepoint passive measurement can be conducted very easily here, compared with two-point passive measurement for measuring the one-way delay.

If we extract the quantity $\sum_{n=1}^{m} 1_{\left\{V\left(T_{n}\right)>c\right\}} / m$ from (3), this quantity is a simple active estimator that counts the packets for the delay to exceeds $c$. However, (3) is weighted by $a\left(T_{n}\right) /\left(\sum_{l=1}^{m} a\left(T_{l}\right) / m\right)$, which is decided by the traffic in the target flow when probe packets arrive. This means that the one-way delay distribution (measured by active measurement without bias) is corrected to the empirical one-way delay distribution by the bias of the target flow (observed by passive measurement). $a\left(T_{n}\right) /\left(\sum_{l=1}^{m} a\left(T_{l}\right) / m\right)$ in (3) corresponds to $\mathrm{d} F_{k}(Y(n)) / \mathrm{d} G(Y(n))$ in (1).

\section{Suboptimal probing}

Since the PASTA property is good for non-biased measurement, Poisson arrivals (intervals with an exponential distribution) have been widely used as policy of probe packets arrivals for active measurement. However, if arrival process of the probe packets is stationary and mixing, under non-intrusive conditions, the following equation holds and we can also ignore the effects of probe packets under nonintrusive conditions.

$$
\lim _{m \rightarrow \infty} \frac{1}{m} \sum_{n=1}^{m} X\left(T_{n}\right)=\mathrm{E}[X(0)] \quad \text { a.s. }
$$

where we assume the target process $X(t)$ is stationary and ergodic. [4] proved (4) and named this property NIMASTA (Non-Intrusive Mixing Arrivals See Time Averages).

The recent study [3] also reported that NIMASTA-based probing is suitable for measurement. That provides an improvement in the accuracy of the measurement. We can 
Table 1. Type of user flows

\begin{tabular}{|c|c|c|c|c|c|}
\hline $\begin{array}{l}\text { Flow } \\
\text { type }\end{array}$ & $\begin{array}{l}\text { Flow } \\
\text { ID }\end{array}$ & $\begin{array}{c}\text { Mean } \\
\text { ON/OFF } \\
\text { period }\end{array}$ & $\begin{array}{l}\text { Distribution of } \\
\text { ON/OFF length }\end{array}$ & $\begin{array}{c}\text { Shape } \\
\text { parameter }\end{array}$ & $\begin{array}{c}\text { Rate at } \\
\text { ON } \\
\text { period }\end{array}$ \\
\hline type 1 & $\# 1-5$ & $10 \mathrm{~s} / 5 \mathrm{~s}$ & Exp & - & $6 \mathrm{Mbps}$ \\
\hline type 2 & \#6-10 & $5 \mathrm{~s} / 10 \mathrm{~s}$ & Exp & - & $6 \mathrm{Mbps}$ \\
\hline type 3 & $\# 11-15$ & $5 \mathrm{~s} / 10 \mathrm{~s}$ & Parete & 1.5 & $9 \mathrm{Mbps}$ \\
\hline type 4 & $\# 16-20$ & $1 \mathrm{~s} / 19 \mathrm{~s}$ & Parete & 1.5 & $9 \mathrm{Mbps}$ \\
\hline
\end{tabular}

Table 2. Type of probing

\begin{tabular}{ccc}
\hline $\begin{array}{c}\text { Distribution of } \\
\text { probe intervals }\end{array}$ & $\begin{array}{c}\text { Parameter of Gamma } \\
\text { distribution }\end{array}$ & $\begin{array}{c}\text { Mean probe } \\
\text { intervals }\end{array}$ \\
\hline Exp & $(\beta=1)$ & $0.5 \mathrm{~s}$ \\
Gamma & $\beta=5$ & $0.5 \mathrm{~s}$ \\
Gamma & $\beta=25$ & $0.5 \mathrm{~s}$ \\
Gamma & $\beta=125$ & $0.5 \mathrm{~s}$ \\
Periodic & $(\beta \rightarrow \infty)$ & $0.5 \mathrm{~s}$ \\
\hline
\end{tabular}

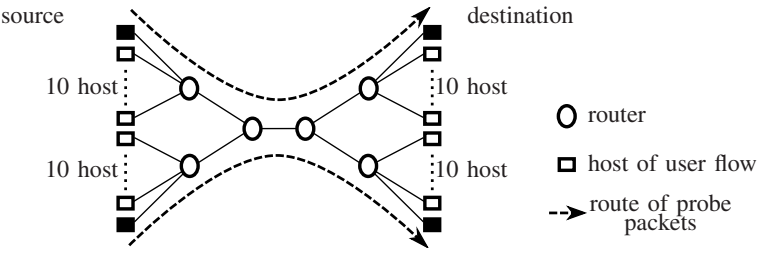

Figure 1. Network model

of the target process $X(t)$ is convex. It can be proven that under the foregoing assumptions, no other probing process with an average interval of $\mu$ has a variance that is lower than that of periodic-probing with determinate intervals (see [3]). A lower variance of the estimator is connected with accuracy. Therefore, periodic-probing is the best policy if we focus only on variance.

On the other hand, periodic-probing does not satisfy the assumptions of NIMASTA due to non-mixing, so periodicprobing is not necessarily the best. This is because a phaselock phenomenon may occur and the estimator may converge on a false value when the cycle of the target process corresponds to the cycle of the probing process.

To tune the tradeoff between traditional policies obeying Poisson arrivals and periodic-probing, [3] proposes a suboptimal policy that gives an inter-probe time that obeys the parameterized Gamma distribution. The probability density function that is used as an inter-probe time is given by

$$
g(x)=\frac{x^{\beta-1}}{\Gamma(\beta)}\left(\frac{\beta}{\mu}\right)^{\beta} \mathrm{e}^{-x \beta / \mu} \quad(x>0),
$$

where $g(x)$ is the Gamma distribution whose shape and scale parameters are $\beta$ and $\mu / \beta$, respectively. $\mu$ denotes the mean, and $\beta$ is the parameter. When $\beta=1, g(x)$ reduces to the exponential distribution with mean $\mu$. When $\beta \rightarrow \infty$, the policy reduces to periodic-probing because $g(x)$ converges on $\delta(x-\mu)$.

If the autocovariance function is convex, it is proven that the variance of estimator $\hat{p}$ sampled by intervals according to (5) monotonically decreases with $\beta$. We can achieve nearoptimal variance of periodic-probing, since (5) corresponds to periodic-probing towards limit $\beta \rightarrow \infty$. The problem of incorrectness due to phase-lock phenomenon can be avoided if we tune $\beta$ to a limited value (a probing process that has intervals as set by (5) is mixing). Solving the tradeoff between a traditional policy obeying Poisson arrivals and periodic-probing, we can get a suboptimal probing process if we give $\beta$ an appropriate value.

\section{The effectiveness of suboptimal probing}

We investigated the effectiveness of Gamma-probing in the framework of CoMPACT monitor, through NS-2 based simulations. The network model is shown in Figure 1.

There are 20 pairs of source and destination end hosts. Each source end host transfers packets by UDP to the corresponding destination end host. User flows are given as ON/OFF processes and categorized into the four types listed in Table 1, with there are five flows in each type.

Probe packet trains are categorized into the five types listed in Table 2. 300 trains of each type are streamed on the two routes shown in Figure 1, so the total number of probe packet trains in the network is 3000 . To analyze the variance of the estimator, we streamed a large number of probe packet trains. Note that parameters of Exp and periodic in Table 2 are parameters of the Gamma distribution corresponding to each probing.

User flow packets and probe packets are 1500 bytes and 64 bytes, respectively. Link capacities are identical at 64 Mbps. No loss occures, since there is sufficient buffering. We ran the simulation for $500 \mathrm{~s}$. The non-intrusive requirement was satisfied (the effect of probe packets can be ignored), since the ratio of the probe stream to all streams is about $0.00197 \%$. We observed the traffic by passive measurement of queue for the edge router on the source side.

First, we show that CoMPACT monitor can estimate the empirical one-way delay when using Gamma-probing. To estimate the complementary CDF of the one-way delay experienced by flow $\# 1$, we use probe packet trains with parameter $\beta=1,25$ and $\beta \rightarrow \infty$ respectively. Each result is shown in Figure 2. Note that the horizontal axis, which is the one-way delay, corresponds to $c$ in (2). To compare the empirical delay with the estimate from CoMPACT monitor, we include the estimate from active measurement in the plot. 

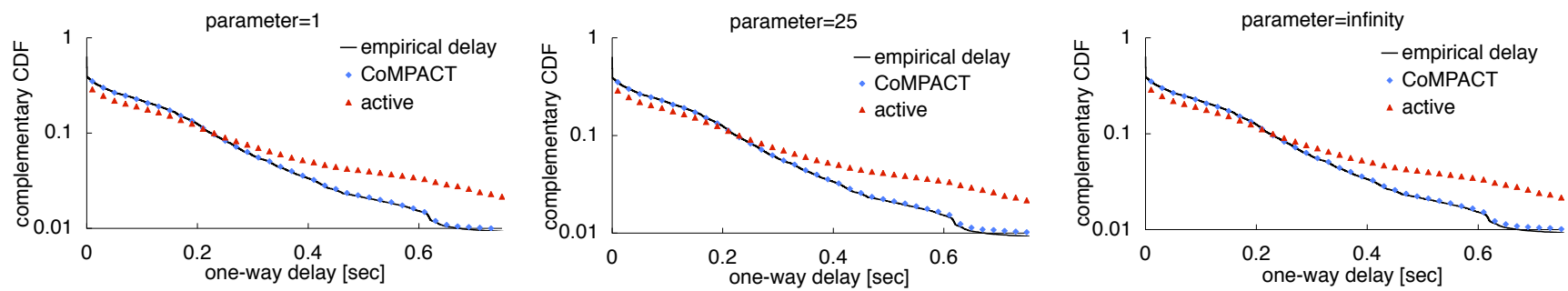

Figure 2. The estimation of comprementary CDF (flow \#1)

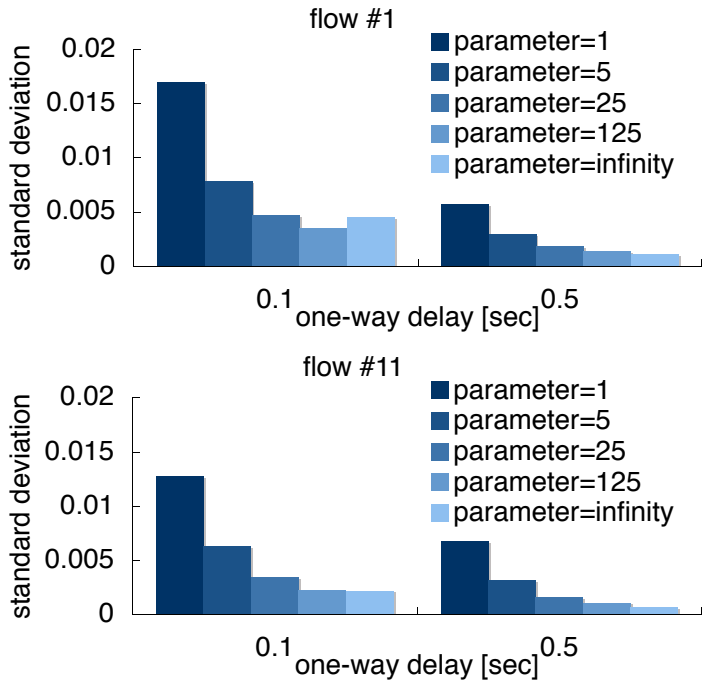

Figure 3. Standard deviation of estimator

In Figure 2, we can see that the CoMPACT monitor gives good estimates of the true value. We cannot judge the superiority or inferiority of any type of probe packet trains. To represent each flow type, we have plotted for flows \#6, $\# 11$ and \#16, getting results similar to Figure 2.

Second, we verify the relationship between the parameter of Gamma distribution that is used as the inter-probe time, and the variance of estimator. If we can apply the theory of [3] to CoMPACT monitor, the variance of the estimator decreases as the parameter increases.

We show the standard deviation of estimator (flow \#1 and flow\#11) in Figure 3. The standard deviation clearly decreases as $\beta$ increases from $\beta=1$ to $\beta=125$. In result of flow \#1 (0.1s), the standard deviation of periodic-probing corresponding to $\beta \rightarrow \infty$ is larger than that for $\beta=125$. This reversal may be a sign of incorrectness due to the phase-lock that occurs when the cycle of the target process corresponds to the cycle of the probing process.

Consequently, for the case of UDP flow, it is confirmed that we can obtain adequate accuracy with a suboptimal probing process if we tune the parameter of Gamma distribution that we use as the inter-probe time.

\section{Conclusion}

In a non-intrusive context where the effect of probe packets can be ignored, it was confirmed that the accuracy of estimating the complementary CDF of one-way delay can be improved by using Gamma-probing as part of applying CoMPACT monitor estimates.

\section{Acknowledgements}

A part of this research was made possible by the Grant-inAid for Scientific Research (B) No. 21300027 (2009- 2011) from the Japan Society for the Promotion of Science.

\section{References}

[1] M. Aida, N. Miyoshi, and K. Ishibashi, "A scalable and lightweight QoS monitoring technique combining passive and active approaches-On the mathematical formulation of compact monitor," in Proc. IEEE INFOCOM 2003, San Francisco, CA, Apr. 2003, pp. 125-133.

[2] M. Aida, N. Miyoshi and K. Ishibashi, "A change-of-measure approach to per-flow delay measurement systems combining passive and active methods: On the mathematical formulation of CoMPACT monitor," IEEE Transactions on Information Theory, vol. 54, no. 11, pp. 4966-4979, Nov. 2008.

[3] F. Baccelli, S. Machiraju, D. Veitch, and J. Bolot, "On optimal probing for delay and loss measurement," in Proc. Int. Measurement Conf. '07, San Diego, CA, Oct. 2007, pp. 291-302

[4] F. Baccelli, S. Machiraju, D. Veitch, and J. Bolot, "The role of PASTA in Network Mesurement," in Proc. ACM SIGCOMM 2006, Pisa, Italy, Sep. 2006, pp. 231-242. 